University of Windsor

Scholarship at UWindsor

\title{
Measurements of total cross sections for positron scattering by uracil molecules
}

\author{
E. Surdutovich \\ G. Setzler \\ W.E. Kauppila \\ Steven J. Rehse \\ University of Windsor
}

Follow this and additional works at: https://scholar.uwindsor.ca/physicspub

Part of the Physics Commons

\section{Recommended Citation}

Surdutovich, E.; Setzler, G.; Kauppila, W.E.; and Rehse, Steven J.. (2008). Measurements of total cross sections for positron scattering by uracil molecules. Physical Review A - Atomic, Molecular, and Optical Physics, 77 (5).

https://scholar.uwindsor.ca/physicspub/23

This Article is brought to you for free and open access by the Department of Physics at Scholarship at UWindsor. It has been accepted for inclusion in Physics Publications by an authorized administrator of Scholarship at UWindsor. For more information, please contact scholarship@uwindsor.ca. 


\title{
Measurements of total cross sections for positron scattering by uracil molecules
}

\author{
E. Surdutovich, ${ }^{1,2}$ G. Setzler, ${ }^{1}$ W. E. Kauppila, ${ }^{1}$ S. J. Rehse, ${ }^{1}$ and T. S. Stein ${ }^{1}$ \\ ${ }^{1}$ Department of Physics and Astronomy, Wayne State University, Detroit, Michigan 48202, USA \\ ${ }^{2}$ Department of Physics, Oakland University, Rochester, Michigan 48309, USA
}

(Received 4 April 2008; published 8 May 2008)

\begin{abstract}
We present measurements of total cross sections for 1 to $30 \mathrm{eV}$ positrons scattered by uracil molecules. The measurements are done using a beam transmission technique on a setup adjusted to accommodate the nature of uracil and we discuss possibilities of future experiments studying positron interactions with more complicated targets. These and future investigations of positron interactions with biomolecules have potential relevance to various biomedical applications.
\end{abstract}

DOI: 10.1103/PhysRevA.77.054701

PACS number(s): 34.80.Uv, 33.15.-e, 87.15.H-

\section{INTRODUCTION}

Positron emission tomography (PET) has paved the road for positron use in the biomedical realm. Even though PET has become common, there are many unanswered questions about interactions of positrons with biomolecules; e.g., positronium formation still remains a "subtle aspect of PET" [1]. This is just one reason why the investigation of the interactions of positrons and biological molecules may be interesting. Another reason comes from a different side of the scientific community, which is interested in studies of DNA and proteins; in that aspect positrons serve as a tool that may be quite attractive. Such a study will have to analyze the products of DNA or protein decay as is done for electron [2] or heavy-ion [3] projectiles. In order to launch this study, a desirable first step is the measurement of cross sections of different types of interactions of positrons with DNA or proteins. However, even this cannot be done without preliminary studies. The exploration of much smaller and simpler molecules such as uracil (one of RNA bases) will hopefully lead to studies of more complex targets. In fact, uracil molecules were one of the first targets for both experimental and theoretical investigations of electron scattering by biomolecules [4-8].

In this work, our measurements of the total scattering cross sections for 1 to $30 \mathrm{eV}$ positrons scattered by uracil molecules are made with a beam-transmission technique, previously used to measure total cross sections of positrons scattered by alkali-metal atoms [9-12], and a variety of room-temperature gases [13]. The setup has been modified to accommodate the nature of the target and these modifications, such as heating of adjacent apertures, will be used in the future experiments with more challenging targets.

\section{MEASUREMENTS AND DISCUSSION}

Our setup for measurements of total cross sections is described in detail most recently in Ref. [12] and references therein. Briefly, we use a ${ }^{22} \mathrm{Na}$-positron source with a tungsten moderator that slows and narrows the energy distribution of the positrons (the full width at half-maximum gradually increases from $0.3 \mathrm{eV}$ for $1-\mathrm{eV}$ positrons to $2 \mathrm{eV}$ for $30-\mathrm{eV}$ positrons), which are then focused by an electrostatic lens and collimated by an axial magnetic field that eventually guides the beam through the scattering cell that may or may not be filled with uracil vapor. Then, the transmitted positrons are detected by a channeltron after passing through a retarding element. If the scattering cell is not heated, the number density of the uracil vapor is small and the attenuation of the beam is negligible. We refer to such a beam as the primary beam to distinguish it from the attenuated beam in the case when the beam interacts with the uracil vapor produced by heating the scattering cell and an adjacent cylinder containing uracil powder. We measure the temperature of the cell and calculate the pressure of the saturated vapor of the sublimated uracil using available vapor pressure data [14]. Having measured the luminosities of the primary and attenuated beams and having calculated the number density of the uracil vapor, we may calculate the total cross section of the positrons scattered by uracil. An ideal total cross section measurement discriminates against all positrons that have interacted with uracil, both elastically or inelastically by removing all scattered positrons from the attenuated beam. In our system, the exit aperture of the scattering cell and the use of a retarding potential applied to the cylindrical element following the scattering cell provide a reasonably high degree of discrimination against positrons that have scattered elastically or inelastically.

Our first attempt to measure cross sections of positrons scattered by uracil failed. The vapor of uracil molecules within the cell diffused through the aperture and coated the cold surrounding surfaces including the surface of the retarding element. As soon as this had happened, the dielectric film would quickly charge up and attenuate the beam much more effectively than the vapor in the cell. This problem has been solved by heating the retarding element by a specially designed heating wire and by additional heating of the whole chamber from the outside by nearly boiling water. Since the operating temperatures were between $130{ }^{\circ} \mathrm{C}$ and $140{ }^{\circ} \mathrm{C}$, this turned out to be sufficient, and we obtained reproducible results.

Our results are presented in Fig. 1 and Table I. These results are the product of a considerable number of measurements. The variance of cross sections at each measured energy is quite significant. Therefore, we were concerned about whether this variance was caused by the difference in the vapor pressure or additional attenuation caused by the reduced but still present uracil coating of surfaces adjacent to the beam. To answer this concern, we have analyzed the 


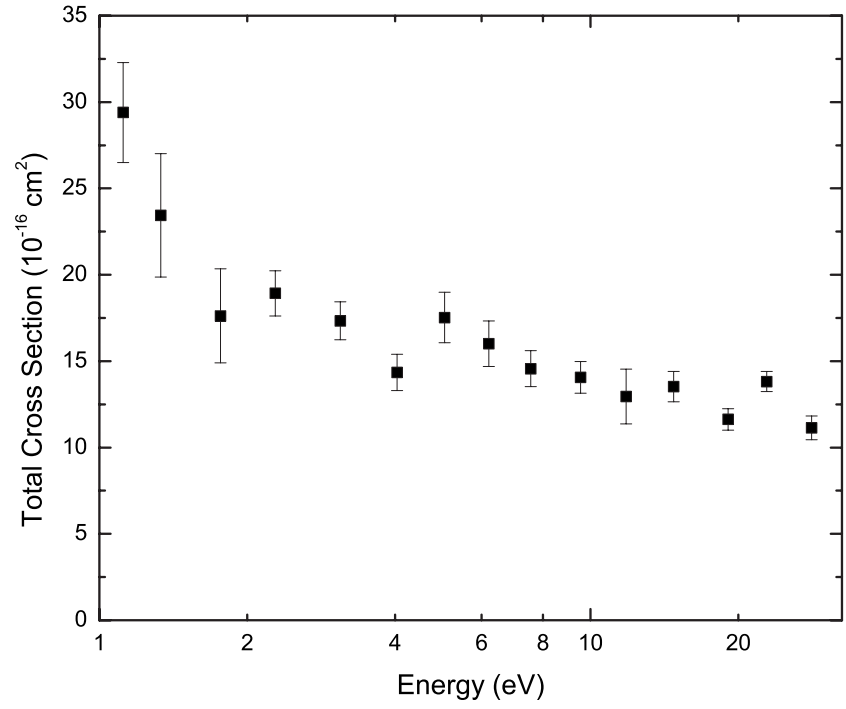

FIG. 1. Total cross sections of positrons scattered by uracil molecules. The error bars indicate the combinations of statistical and part of systematic uncertainties.

statistical uncertainty of each point and looked at how this uncertainty correlated with the difference of this point from the average cross section. The results of this analysis showed no correlation. The averaging was done in two ways, by simple calculation of the average at a given energy and by calculation of the weighted average using the inverse squares of uncertainties as weights. Both averages turned out to be very close to each other.

As far as we know, there are no theoretical calculations of positron-uracil cross sections at this time. Comparison to electrons scattered by uracil does not seem to be productive at the measured energies since the processes that may contribute significantly at these energies, such as positronium formation for positrons and dissociative attachment for electrons are quite different. (The energies at which our measurements have been done are much lower than the energies at which we would expect the total cross sections of electrons and positrons to asymptotically approach each other.) The positron-uracil cross sections are not particularly large (approaching $30 \AA^{2}$ near $1 \mathrm{eV}$ ), but are comparable to polyatomic hydrocarbons [13]. The apparent change in slope of the total cross section curve in the vicinity of $2 \mathrm{eV}$ suggests that positronium formation may be a significant contributor to total scattering above the positronium formation threshold near $2.5 \mathrm{eV}$, as has been found for most target atoms and molecules that have been investigated [13].
TABLE I. Total cross sections in $e^{+}$-uracil scattering with statistical and part of systematic uncertainties (in parentheses) with respect to $e^{+}$energy.

\begin{tabular}{lc}
\hline \hline Energy $(\mathrm{eV})$ & $e^{+}$-uracil $Q_{T}(\AA)^{2}$ \\
\hline 1.1 & $29.4(2.9)$ \\
1.3 & $23.4(3.6)$ \\
1.8 & $17.6(2.7)$ \\
2.3 & $18.9(1.3)$ \\
3.1 & $17.3(1.1)$ \\
4.0 & $14.4(1.1)$ \\
5.0 & $17.5(1.5)$ \\
6.2 & $16.0(1.3)$ \\
7.6 & $14.6(1.0)$ \\
9.5 & $14.1(0.9)$ \\
11.8 & $13.0(1.6)$ \\
14.8 & $13.5(0.9)$ \\
19.0 & $11.6(0.6)$ \\
22.9 & $13.8(0.6)$ \\
28.2 & $11.1(0.7)$ \\
\hline \hline
\end{tabular}

\section{CONCLUSIONS}

In conclusion, we would like to speculate about the possibility of further measurements of positron scattering from biomolecules. From our experience with uracil, we infer that such measurements are possible, although they may not be simple. The "nonsticking" temperature may have to be higher even in the case of other DNA or RNA bases. This will be related to some technical limitations of the existing apparatus. The other problem which will have to be solved is related to the products of the decay of the target molecules as a result of their dissociation, such as the strategic fragmentation of peptides. This requires implementations of massspectrometry by means of, e.g., time-of-flight measurement in a style of Ref. [3]. This work shows that the problems caused by the capricious nature of biomolecules can be solved.

\section{ACKNOWLEDGMENTS}

This work was partly supported by NSF Grant No. PHY99-88093 and NSF-REU Grant No. EEC-0552772. We are grateful to P. Papoulias, J. S. Payson, and A. Sebastian for fruitful discussions.
[1] M. D. Harpen, Med. Phys. 31, 57 (2004).

[2] K. Aflatooni, A. M. Scheer, and P. D. Burrow, Chem. Phys. Lett. 408, 426 (2005).

[3] J. de Vries, R. Hoekstra, R. Morgenstern, and T. Schlathölter, Phys. Rev. Lett. 91, 053401 (2003).

[4] G. Hanel, B. Gstir, S. Denifl, P. Scheier, M. Probst, B. Farizon,
M. Farizon, E. Illenberger, and T. D. Märk, Phys. Rev. Lett. 90, 188104 (2003).

[5] S. Denifl, B. Sonnweber, G. Hanel, P. Scheier, and T. D. Märk, Int. J. Mass Spectrom. 238, 47 (2004).

[6] A. M. Scheer, K. Aflatooni, G. A. Gallup, and P. D. Burrow, Phys. Rev. Lett. 92, 068102 (2004). 
[7] N. Russo, M. Toscano, and A. Grand, J. Comput. Chem. 21, 1243 (2000).

[8] P. Mozejko and L. Sanche, Radiat. Environ. Biophys. 42, 201 (2003).

[9] C. K. Kwan, W. E. Kauppila, R. A. Lukaszew, S. P. Parikh, T. S. Stein, Y. J. Wan, and M. S. Dababneh, Phys. Rev. A 44, 1620 (1991).

[10] W. E. Kauppila, C. K. Kwan, T. S. Stein, and S. Zhou, J. Phys. B 27, L551 (1994).

[11] S. P. Parikh, W. E. Kauppila, C. K. Kwan, R. A. Lukaszew, D.
Przybyla, T. S. Stein, and S. Zhou, Phys. Rev. A 47, 1535 (1993).

[12] E. Surdutovich, W. E. Kauppila, C. K. Kwan, E. G. Miller, S. P. Parikh, K. A. Price, and T. S. Stein, Phys. Rev. A 75, 032720 (2007).

[13] W. E. Kauppila and T. S. Stein, Adv. At., Mol., Opt. Phys. 26, 1 (1990).

[14] B. Brunetti, V. Piacente, and G. Portalone, J. Chem. Eng. Data 45, 242 (2000). 